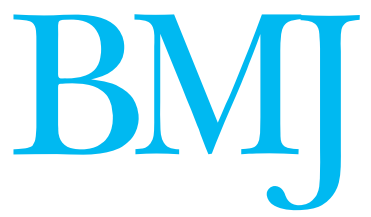

\title{
Treating depression in later life
}

\author{
We need to implement the evidence that exists
}

$\mathrm{T}$ The three major mental health problems affecting older people are dementia, delirium, and depression. Depressive disorders are the most common, affecting one in seven, a prevalence rate that is consistent across countries and cultures. ${ }^{1}$ Comparable rates for dementia and delirium are one in 17 and one in 25. Therapeutic optimism surrounds the management of dementia and delirium, but the development of new management approaches in late life depression has been neglected, perhaps because of stigma. Twenty years ago, a rule of three was proposed in this journal: a third of older depressed people get better, a third remain the same, and a third get worse. ${ }^{2}$ This rule is still applicable today despite the availability and efficacy of treatments that could substantially improve these ratios. Depression late in life is associated with serious morbidity and mortality, including suicide. ${ }^{3}$ The national service framework for older people targets stroke, falls, dementia, and depression, but only the first three seem to attract new funding or services commissioned by primary care trusts.

Two thirds of older people with serious depression do not have symptoms that fit current classifications of mood disorders, ${ }^{1}$ which have been generated to reflect symptoms in younger people. Older people may have insufficient symptoms to meet the threshold for a disorder, and presentation differs from younger people because of ageing, physical illness, or both.

Detection of depression is poor, ${ }^{4}$ and primary care providers may lack the necessary consultation skills or confidence to diagnose late life depression correctly. Although this is unproved, they may be wary of opening a Pandora's box in time limited consultations and share therapeutic nihilism with the patient. ${ }^{5}$

The evidence base for the management of late life depression is increasing. The number needed to treat for major depression treated with antidepressants is about four and is similar to other age groups. ${ }^{6}$ Perhaps patients who are referred to old age psychiatry services do better as specialists tend to be more persistent in monitoring treatment and encouraging compliance, but $90 \%$ of older depressed people do not see a specialist. ${ }^{7}$ Older people may be distrustful of tablets, not take them, and in recent research have expressed a preference for psychological interventions. ${ }^{8}$

Such interventions are effective in people with late life depression and anxiety, but such therapeutic options may be unavailable in primary care. ${ }^{9}{ }^{10}$

Studies have evaluated interventions such as dissemination of guidelines, educational packages offered by a nurse to primary care doctors, feedback of screening test results, and recommending that antidepressants are prescribed to patients screened positive for depression. ${ }^{11}$ None of these has been shown to improve outcome in patients. ${ }^{11}$

Innovations in the management of depression have been evaluated. The best results come from models that use multifaceted interventions and principles of collaborative care. These vary but generally are brief and include the deployment of care managers and flexible collaboration between primary and specialist care, to improve access to the psychiatrist and mental health teams for the primary care team. Important new evidence shows that this approach is very effective in older depressed patients. Unützer et al randomised 1801 depressed primary care patients aged over 60 to usual care by their primary care doctor or collaborative care. ${ }^{8}$ Collaborative care was delivered by a depression care manager (a nurse or a psychologist) under the supervision of a psychiatrist and a primary care doctor. The main components of the intervention were case management, education about depression, and management of medication. At 12 months, almost half the patients in the intervention group were at least 50\% improved from baseline, compared with only one in five of those receiving usual care. In addition, patients in the collaborative care group were more satisfied, complied better with treatment, and experienced less functional impairment. The cost of the intervention was about $£ 370$ ( $\$ 670$; €550) per patient over 12 months. This is substantially less than a year's worth of antidementia drugs (about £1000). A similar approach was effective in minor depression and dysthymia. ${ }^{12}$

Keeping the patient well is as important as initial treatment. Antidepressants-both tricyclics and selective serotonin reuptake inhibitors-and psychological interventions prevent relapse, and some patients may benefit particularly from combining these modalities. ${ }^{13}$ Such a need for long term management may necessitate an approach through the chronic disease model.

New challenges arise from evidence showing that a proportion of late onset depression arises because of age associated cerebrovascular disease. ${ }^{7}$ So treatments of the future may include some not usually regarded as part of the psychiatrist's toolkit-for example, drugs to halt vascular damage. In the meantime the task for pri- 
mary care must be to move away from the old model of relying on the passive prescription of pills.

The treatment of depression in late life requires some imaginative commissioning at the interface of primary and secondary care. These arrangements should incorporate the new evidence base, favouring active care management and timely support from specialist mental health services.

Carolyn Chew-Graham senior lecturer in primary care Rusholme Academic Unit, Rusholme Health Centre, Manchester M14 5NP

Robert Baldwin consultant psychiatrist

Manchester Mental Health and Social Care Trust, Manchester Royal Infirmary, Manchester M13 9WL

Alistair Burns professor of old age psychiatry

Education and Research Centre, Wythenshawe Hospital, Manchester M23 9PL

(A_Burns@fs1.with.man.ac.uk)

Competing interests: None declared.

1 Beekman AT, Copeland JR, Prince, MJ. Review of community prevalence of depression in later life. Br J Psychiatry 1999;174:307-11.
Millard PH. Depression in old age. BMJ 1983; 287:375-6.

Baldwin RC, Chiu C, Graham N, Katona CLE. Guidelines for depressive disorder of later life: practising the evidence. London: Martin Dunitz, 2002.

4 Baldwin, R, Anderson, D, Black, S, Evans, S, Jones, S, Wilson, K, et al. Guidelines for the management of late-life depression in primary care. Int J Geriatr Psychiatry 2003;18:829-38.

5 Montano CB. Primary care issues related to the treatment of depression in elderly patients. J Clin Psychiatry 1999;60:45-51.

6 Katona C, Livingston G. How well do antidepressants work in older people? J Affect Disord 2002;69:47-52.

7 Thomas AJ, Kalaria RN, O'Brien JT. depression and vascular disease: Thomas AJ, Kalaria RN, O'Brien JT. depression
what is the relationship? J Affect Dis 2004;79:81-95..

8 Unü is the relationship? Katon W, Callahan CM, Williams JW Jr, Hunkeler E, Harpole $\mathrm{L}$, et al. Collaborative care management of late life depression in the primary care setting. JAMA 2002;288:2836-45.

9 Barrowclough C, King P, Colville J, Russell E, Burns A, Tarrier N. A randomised trial of the effectiveness of cognitive-behavioural therapy and supportive counselling for anxiety symptoms in older adults. J Consult Clinical Psychol 2001;69:756-62.

10 Chew-Graham C, Mullin S, May CR, Hedley S, Cole H. Managing depression in primary care: another example of the inverse care law? Fam Pract 2002;19:632-7.

11 Peveler R, Kendrick T. Treatment delivery and guidelines in primary care Br Med Bull 2001;57:193-206.

12 Ciechanowski P, Wagner E, Schmaling K, Schwartz S, Williams B, Diehr P, et al. Community-integrated home based depression treatment in older adults: a randomised controlled trial JAMA 291;13:1569-77.

13 Reynolds III CF, Frank E, Perel JM. Nortriptyline and interpersonal psychotherapy as maintenance therapies for recurrent major depression a randomized controlled trial in patients older then 59 years. JAMA $1996 ; 281: 39-45$

\section{Which drugs should be available over the counter?}

\section{The criteria are clear and include safety, timeliness, and opportunity cost}

A contraception after intercourse, levonorgestrel is available by prescription in the United States and in most other countries. In over 30 countries it is available without prescription. ${ }^{1}$ Levonorgestrel recently came to wide attention when the US Food and Drug Administration (FDA) acted on an application to switch the drug to non-prescription or "over the counter" status. ${ }^{2}$ The application was supported by essentially all internal scientific staff and the external advisory committee of the FDA, but the FDA rejected the application. The reason given had to do with the ability of women to understand the appropriate use of the product, ${ }^{3}$ but this issue had been explicitly discussed and settled to the satisfaction of the FDA's scientists and external advisory committee. ${ }^{4}$ The FDA's explicit denial that the decision had been the result of political pressure has been received with scepticism. ${ }^{5-8}$

How should policy makers decide which drugs should be available over the counter? Practice varies widely. Travellers from developed countries are often surprised to find that antibiotics, antiarrhythmics, and many other drugs are available without prescription in other parts of the world. Even within the United States, some pharmaceuticals are available over the counter in some states but not in others. ${ }^{9}$

Marketing status is not just a choice between requiring and not requiring prescriptions. Drugs with special risks (for example, some antiarrhythmics) are often given a hyperprescription status and sometimes involve central registers of prescribers and patients. To slow the development of bacterial resistance, some hospitals assign hyperprescription status to selected antibiotics. Teratogenic drugs (such as thalidomide) may be dispensed under hyperprescription rules, requiring periodic certificates of non-pregnancy.

In the middle, some jurisdictions make use of pharmacist mediated ("behind the counter") status for non-prescription drugs whose use requires professional guidance, but not necessarily that of a doctor. Toward the loose end of the scale, a product's labelling may instruct patients not to use the product unless a doctor has made the diagnosis, perhaps during an earlier episode of the disease. Finally, many products are available over the counter with no restrictions.

The legal options are different in different jurisdictions. Still, the pertinent considerations are the same everywhere, and they are easy to enumerate.

\section{Diagnostic considerations}

Over the counter status is unlikely to be awarded to a drug whose only use is for a condition (such as rheumatoid arthritis, choriocarcinoma, ulcerative colitis, systemic lupus, streptococcal pharyngitis, multiple myeloma) whose diagnosis could not reliably be made by the patient, perhaps because it requires special expertise or laboratory work. The patient's diagnostic difficulties might change with time. Patients could not be expected to make the initial diagnosis of diabetes, but thereafter they will generally carry the diagnosis for life, and most forms of insulin are accordingly available over the counter in the United States. A woman with her first episode of vaginitis due to candidiasis is not expected to distinguish it from other vaginitides, but she is trusted in many jurisdictions to recognise recurrences and to purchase antifungal preparations over the counter to treat them. 